\title{
Subdomain structure of the co-chaperone SGTA and activity of its androgen receptor client
}

\author{
Andrew P Trotta ${ }^{1,2}$, Eleanor F Need ${ }^{1}$, Lisa M Butler ${ }^{2}$, Luke A Selth ${ }^{2}$, Melissa A O'Loughlin ${ }^{1}$, \\ Gerhard A Coetzee ${ }^{3}$, Wayne D Tilley ${ }^{2}$ and Grant Buchanan ${ }^{1}$ \\ ${ }^{1}$ Cancer Biology Group, Level 1 Basil Hetzel Institute for Translational Health Research, Freemasons Foundation Centre for Men's Health, Queen Elizabeth Hospital, University of \\ Adelaide, 28 Woodville Road, Woodville South, Adelaide, South Australia 5011, Australia \\ ${ }^{2}$ Dame Roma Mitchell Cancer Research Laboratories, Hanson Institute, University of Adelaide, Adelaide, South Australia, Australia \\ ${ }^{3}$ Departments of Preventive Medicine and Urology, Keck School of Medicine, Norris Cancer Center, University of Southern California, Los Angeles, California, USA
}

(Correspondence should be addressed to G Buchanan; Email: grant.buchanan @ adelaide.edu.au)

\begin{abstract}
Ligand-dependent activity of steroid receptors is affected by tetratricopeptide repeat (TPR)-containing co-chaperones, such as small glutamine-rich tetratricopeptide repeat-containing alpha (SGTA). However, the precise mechanisms by which the predominantly cytoplasmic TPR proteins affect downstream transcriptional outcomes of steroid signaling remain unclear. In this study, we assessed how SGTA affects ligand sensitivity and action of the androgen receptor (AR) using a transactivation profiling approach. Deletion mapping coupled with structural prediction, transcriptional assays, and in vivo regulation of AR-responsive promoters were used to assess the role of SGTA domains in AR responses. At subsaturating ligand concentrations of $\leq 0.1 \mathrm{nM} 5 \alpha$-dihydrotestosterone, SGTA overexpression constricted AR activity by an average of $32 \%(P<0.002)$ across the majority of androgen-responsive loci tested, as well as on endogenous promoters in vivo. The strength of the SGTA effect was associated with the presence or absence of bioinformatically predicated transcription factor motifs at each site. Homodimerizaion of SGTA, which is thought to be necessary for chaperone complex formation, was found to be dependent on the structural integrity of amino acids 1-80, and a core evolutionary conserved peptide within this region (amino acids 21-40) necessary for an effect of SGTA on the activity of both exogenous and endogenous AR. This study provides new insights into the subdomain structure of SGTA and how SGTA acts as a regulator of AR ligand sensitivity. A change in AR:SGTA ratio will impact the cellular and molecular response of prostate cancer cells to maintain androgenic signals, which may influence tumor progression.
\end{abstract}

Journal of Molecular Endocrinology (2012) 49, 57-68

\section{Introduction}

The unliganded androgen receptor (apo-AR) in normal cells is located predominately in the cytoplasm in association with heat-shock protein 70 (HSP70) and/or HSP90 chaperone complexes (Marcelli et al. 2006). Although the interaction with chaperone complexes may facilitate folding of the nascent AR protein, ligand binding, and transport (Thomas et al. 2006), the biological necessity for active exclusion of the apo-AR from the nucleus remains unclear. One possibility is that the process of nuclear shuttling allows the cytoplasmic signaling environment to influence transcriptional responses in the nucleus (Ziegler \& Ghosh 2005, Sun et al. 2007). A redistribution of AR subcellular localization in disease states may therefore be indicative of an altered status of receptor activation or response. This is most commonly observed in prostate cancer following the failure of androgen deprivation therapy, whereby the AR subcellular distribution shifts to become predominately nuclear (Zhang et al. 2003). Understanding how the AR translocates to the nucleus, and the role of the chaperone complex in this process, is therefore imperative for our overall appreciation of AR action in both normal development and cancer.

HSP90 forms the core of the steroid receptor chaperone complex but by itself is unable to interact with steroid receptors without one of a set of accessory tetratricopeptide repeat (TPR)-containing co-chaperones that include immunophilin FK506 binding proteins (FKBP) 4 and 5, cyclophilin 40 (CYP40 (CYP27B1)), and phosphatase protein 5 (PP5 (PPP5C)) (Pratt \& Toft 1997). Although each TPR co-chaperone has a different cellular function, their relative abundance affects HSP90-directed ligand binding, localization, and transcriptional activity of different steroid receptors (Barent et al. 1998, Cheung-Flynn et al. 2005, Yong et al. 2007, Schulke et al. 2010). The importance of the TPR molecules in these processes is exemplified by severe defects in predominantly androgen-sensitive tissues in Fkbp4 knockout mice and by high endogenous levels of FKBP5 that correlate with glucocorticoid resistance in squirrel monkeys 
(Denny et al. 2000, Cheung-Flynn et al. 2005, Yong et al. 2007). Furthermore, in prostate cancer, both FKBP5 and CYP40 appear to be required for continued AR activity in an androgen-depleted environment (Periyasamy et al. 2010). Together, these results infer a requirement for the TPR co-chaperones in maintaining normal steroid receptor signaling.

We have previously demonstrated that the TPR co-chaperone small glutamine-rich tetratricopeptide repeat-containing alpha (SGTA) is a binding partner of the AR that acts to exclude the receptor from the nucleus at ligand concentrations below a threshold value (Buchanan et al. 2007). The outcome of SGTAmediated nuclear exclusion is a decrease in AR transactivation activity. However, this affect has only been reported on the minimal synthetic androgenresponsive probasin promoter (ARR3; Buchanan et al. 2007) and it is unknown whether the actions of SGTA on AR activity are ubiquitous throughout the human genome or specific to a set of AR-responsive loci. In addition to its expression in a wide variety of cells and tissues including the heart, brain, lung, liver, muscle, testis, and prostate, SGTA has been reported to play a role in mammalian cellular division, apoptosis, and gene regulation (Cziepluch et al. 1998, 2000, Winnefeld et al. 2004, 2006, Yin et al. 2006, Buchanan et al. 2007). Furthermore, the observation of increased AR nuclear localization with prostate cancer progression is concomitant with a decline in SGTA levels (Buchanan et al. 2007).

Consistent with a role in steroid receptor action, the sequence of the central TPR of SGTA is more closely related to an analogous domain of PP5, FKBP4, and FKBP5 than to any of the more than 100 other TPR-containing proteins that are not implicated in steroid receptor signaling (Buchanan et al. 2007), inferring a specific role for SGTA in AR action. Structurally, SGTA contains three recognized domains: a centralized TPR involved in interactions with HSP90, HSP70, growth hormone receptor (GHR), and myostatin; a glutamine-rich carboxyl terminus (QRD) shown to interact with cysteine string protein and thereby alter synaptic transmission (Cziepluch et al. 1998, Angeletti et al. 2002, Schantl et al. 2003, Tobaben et al. 2003, Wang et al. 2003, Liou \& Wang 2005); and an amino terminal region implicated in SGTA homodimerization, which may act to inhibit the interaction between the TPR domain and GHR (Schantl et al. 2003). Although SGTA remains the only known steroid receptor-associated TPR co-chaperone to form a homodimer, the regions of SGTA necessary for homodimerization as well as the biological requirement for SGTA homodimerization remain elusive. Moreover, given that HSP90 is thought to generate only a single TPR binding site per steroid receptor complex, the role of SGTA homodimerization in its regulation of AR function is of particular interest (Pratt \& Toft 1997).
The overall objectives of this study were to determine whether the effect of SGTA on AR activity remained consistent across a number of androgen-responsive loci and to investigate the functional relationship between SGTA homodimerization and AR activity. This study further aimed to better understand the biological necessity and flexibility of SGTA homodimerization as a potential approach to modulate AR signaling.

\section{Materials and methods}

\section{Plasmids}

Expression plasmids pCMV-AR3.1, pM-SGTA, and pVP16-SGTA, pBS-SK; androgen-responsive reporters ARR3-tk-Luc, R01, R12, R16, R20, R22, R24, R26, R35, R42, R61, and R62; and the GAL4-DBD-responsive pGK-1 reporter have been described previously (Buchanan et al. 2004a, 2007, Butler et al. 2006, Jia et al. 2008, Need et al. 2009). Primer-overlap extension (Buchanan et al. 2004b, Need et al. 2009) was used to respectively generate SGTA deletions in $\mathrm{pM}$ and pVP16 expression plasmids. The pcFH expression plasmid, a derivative of pCDNA3.1 (Life Technologies), was engineered to encode a dual FLAG and HA epitope at the amino terminus of any cloned sequence using an oligonucleotide cloning approach. Oligonucleotide primers and restriction endonucleases used to create plasmids for this study are listed.

\section{Cell culture}

COS-1 cells (American Type Culture Collection, Rockville, MD, USA) and the PC3 subline PC3 ${ }^{\mathrm{AR}+}$ described in Buchanan et al. (2004a) were maintained in RPMI-1640 supplemented with 5\% fetal bovine serum (FBS; Sigma-Aldrich). LNCaP cells (American Type Culture Collection) were maintained in RPMI$1640+10 \%$ FBS. PC3 ARR3-Luc and PC3 pGL4.14 stable cell lines were created and maintained as described previously (Need et al. 2009). All assays requiring steroids were performed in phenol red-free RPMI-1640 (Sigma-Aldrich) with 5\% dextran-coated charcoal-stripped FBS.

\section{Mammalian two-hybrid assays and immunoblot analysis}

COS-1 cells (20 000 cells/well in 96-well plates) were transfected using Lipofectamine 2000 (Life Technologies) with $0 \cdot 1-20 \mathrm{ng}$ of each of GAL4-DBD and VP16-AD fusion plasmids expressing SGTA or its fragments and $25 \mathrm{ng}$ pGK1 reporter. In all experiments, the molar amounts of each expression/control plasmid were kept constant, and total DNA per well was 
maintained by the addition of prokaryotic expression plasmid pBS-SK + . Luciferase activity was analyzed $24 \mathrm{~h}$ after treatment as described previously (Buchanan $\mathrm{et} \mathrm{al}$. 2007 ) and is presented as mean ( \pm s.E.M.) of six independently transfected wells for each plasmid combination and experimental condition. All data represent experiments performed independently at least three times. Where indicated, lysates from replicates were pooled and equal volumes resolved on SDS-PAGE and immunoblotted using SGTA (Abnova, Taipei City, Taiwan), HA, and GAL4-DBD (Santa Cruz Biotechnology) antisera, as described previously (Buchanan et al. 2007, Need et al. 2009).

\section{SGTA overexpression for SDS/nondenaturing PAGE and RT-PCR}

LNCaP cells and COS- 1 cells $\left(2 \cdot 5 \times 10^{5}\right.$ cells/well in sixwell plates) were transfected using Lipofectamine 2000 (Life Technologies) with $500 \mathrm{ng}$ pCFH, pCFH-SGTA, pCFH-SGTA $\Delta 1-20$, pCFH-SGTA $\Delta 21-40$, pCFHSGTA $\Delta 41-60$, pCFH-SGTA $\Delta 29-33$, pCFH-SGTA 80-313, pCFH-SGTA $\Delta$ 91-192, pCFH-SGTA $\Delta 200-263$, or pCFH-SGTA 1-270 for $24 \mathrm{~h}$. Cells treated with vehicle (ethanol) control or $0 \cdot 1-1 \mathrm{nM} 5 \alpha$-dihydrotestosterone (DHT) for $16 \mathrm{~h}$ were indicated. Protein was harvested and resolved on either an SDS-PAGE or nondenaturing PAGE gel and immunoblotted using SGTA antisera. RNA was extracted using RNeasy RNA isolation kit (Qiagen) according to the manufacturer's instructions. Synthesis of cDNA was performed on $1 \mu \mathrm{g}$ RNA using the iScript cDNA synthesis kit (Bio-Rad) according to the manufacturer's instructions. For all RT-PCRs, $1 \mu \mathrm{l}$ cDNA was used with $2 \times$ Iq SYBR Green Mastermix (Bio-Rad) and 5 pmol of sense and antisense primers listed. The housekeeper gene GAPDH was used as a normalization control. The following cycling parameters were used for all reactions: $95^{\circ} \mathrm{C}$ for $3 \mathrm{~min}$ followed by 40 cycles of $95^{\circ} \mathrm{C}$ for $15 \mathrm{~s}, 55^{\circ} \mathrm{C}$ for $15 \mathrm{~s}$, and $72^{\circ} \mathrm{C}$ for $30 \mathrm{~s}$ and a melt curve performed from 55 to $95^{\circ} \mathrm{C}$ at $0.5^{\circ} \mathrm{C}$ for $10 \mathrm{~s}$.

\section{Secondary structure prediction, residue composition, and logo creation}

The nnpredict online server (www.cmpharm.ucsf.edu/ $\sim$ nomi/nnpredict.html; Katzman et al. 2008) was used to define potential alpha helical and beta sheet structural elements in human SGTA. Serine, threonine, and tyrosine amino acid phosphorylation prediction was performed using the NetPhos 2.0 online server (http://www.cbs.dtu.dk/services/NetPhos/; Blom et al. 1999). Amino acid composition analysis was performed using Composition Predictor (www.cprofiler.org/) with the number of bootstrap iterations set at 10000 and
$P$ value $<0.05$ (Vacic et al. 2007). Sequence logos were created using Weblogo (http://weblogo.berkeley.edu/).

\section{Transcription factor motif search}

AR-occupied regions (ARORs) were scanned for known transcription factor motifs from the JASPAR CORE vertebrata database (Bryne et al. 2008) using default parameters in CisGenome (Ji et al. 2008). Fold enrichment and significance (Fisher's exact test) were estimated by comparing the frequency of motif occurrence in the ARORs to the frequency of motif occurrence in fifty $1 \mathrm{~kb}$ negative control genomic regions. The control genomic regions were chosen to match the physical distribution of the ARORs (Ji et al. 2008). Only motifs that were significantly enriched $(P \leq 0 \cdot 01)$ are reported.

\section{AR transactivation assays}

PC3 cells (10 000 cells/well in a 96-well plate) were transfected with $2.5 \mathrm{ng}$ pCMVAR-3.1, $5 \mathrm{ng}$ pcFH-SGTA, pCFH-SGTA deletion variants (Fig. 3A) or equivalent molar amount of empty pcFH, and $50 \mathrm{ng}$ of an AR-responsive-luciferase reporter/well. Cells were transfected and treated with vehicle (ethanol) control or increasing concentrations of DHT for $24 \mathrm{~h}$ in replicates of six and assayed for luciferase activity. Data were combined from two to five independent transactivation experiments for each reporter (up to 30 biological replicates/reporter).

\section{Statistical analyses}

Statistical analyses were performed as indicated in figure legends using GraphPad Prism Version 5 Software for Windows (GraphPad Software, La Jolla, CA, USA). Where there were only two comparisons, the MannWhitney $U$ test was performed. The Kruskal-Wallis and Dunn's multiple comparison post hoc test was used to compare three or more groups. The Spearman $\rho$ test was used to correlate differences between variables.

\section{Results}

\section{SGTA affects AR sensitivity to ligand at multiple transcriptionally responsive loci}

Our previous studies demonstrated that ectopic expression of SGTA diminished AR sensitivity to DHT on the minimal synthetic androgen-responsive probasin promoter (ARR3; Buchanan et al. 2007). To determine whether the effect of SGTA applies more broadly to AR transcriptional function, we used a set of 13 AR-responsive luciferase reporters derived from 
our investigation of the AR cistrome on chromosomes 20 and 21 (Jia et al. 2008). The genomic locations for all the AR binding loci used in this study are listed in Table 1. SGTA overexpression decreased basal activity of $\sim 50 \%$ of the reporters $(7 / 13 ; P<0.05$; Fig. 1A) and a similar proportion $(6 / 13)$ following treatment with $0.01 \mathrm{nM}$ DHT $(P<0.05$; Fig. 1B). Markedly, however, SGTA overexpression repressed ligand-dependent AR activity induced by $0 \cdot 1 \mathrm{nM}$ DHT at $12 / 13$ reporters tested $(P<0.05$; Fig. 1C) but was far less effective at higher ligand concentrations (Fig. 1D). Using PC3 cells stably integrated with the probasin ARR3 reporter, we were able to demonstrate that the DHT concentrationdependent repression of AR by SGTA is maintained in a chromatin context $(P<0 \cdot 05$; Fig. $1 \mathrm{E})$.

Although SGTA repressed AR activity at $0 \cdot 1 \mathrm{nM}$ DHT on most reporters, the magnitude of repression appeared to depend on the specific promoter (Fig. 1A, B, C and D). For example, SGTA significantly decreased AR activity on the R22 reporter at all ligand concentrations $(P<0.05)$ but did not affect R35 at any (Fig. 1A, B, C and D). Comparing reporters, we identified a positive correlation between the strength of the nascent AR response to DHT and the extent to which SGTA overexpression repressed AR activity on that promoter at $0 \cdot 1 \mathrm{nM}$ DHT $(P<0 \cdot 006$; Fig. 1F). In other words, the more responsive the loci to AR, the greater the effect of SGTA in decreasing AR activation of it. Overall, 72\% of SGTA inhibition can be ascribed to the level of DHT responsiveness. It is important to note that the difference in nascent AR response of those reporters strongly affected by SGTA (e.g. ARR3 and R01) vs those weakly affected (e.g. R24, R35, R42, and R62) is consistent at all ligand concentrations, demonstrating that the effect of SGTA is not due to a shifted ligand sensitivity of each promoter.

Table 1 List of nucleotide start and end sites for each androgen receptor (AR) binding site on chromosomes 19 and 20 that were subcloned into a TK-luciferase reporter construct

\begin{tabular}{|c|c|c|c|}
\hline \multirow[b]{2}{*}{$\begin{array}{l}\text { AR-responsive } \\
\text { eporter }\end{array}$} & \multirow[b]{2}{*}{ Chromosome } & \multicolumn{2}{|c|}{$\begin{array}{l}\text { Chromosomal fragment } \\
\text { characteristics }\end{array}$} \\
\hline & & $\begin{array}{l}\text { Nucleotide } \\
\text { start site }\end{array}$ & $\begin{array}{l}\text { Nucleotide } \\
\text { end site }\end{array}$ \\
\hline 1 & 19 & 18669693 & 18670951 \\
\hline 12 & 19 & 46118758 & 46119343 \\
\hline 16 & 20 & 5028554 & 5029092 \\
\hline 20 & 20 & 9263576 & 9264058 \\
\hline 22 & 20 & 10790223 & 10790772 \\
\hline 24 & 20 & 12875368 & 12875768 \\
\hline 26 & 20 & 19893130 & 19893681 \\
\hline 335 & 20 & 31663395 & 31663891 \\
\hline 242 & 20 & 36666768 & 36667339 \\
\hline 244 & 20 & 38530852 & 38531435 \\
\hline 261 & 20 & 31783934 & 31784525 \\
\hline 362 & 19 & 56045778 & 56046295 \\
\hline
\end{tabular}

To investigate what might determine a locus-specific effect of SGTA on AR activity, we dichotomized the native AR reporters (i.e. excluding the minimal ARR3 probasin reporter) into those on which AR activity was strongly affected by SGTA $(>25 \%$ decrease, five reporters) vs those affected weakly $(<25 \%$, seven reporters) and undertook bioinformatic analysis for enriched transcription factor motifs. As a comparison, we used 50 randomly chosen and distribution-matched control genomic regions. Of the seven motifs enriched, four (FOXA1, FOXA2, AR, and FOXO3) were threefold or more enriched in both reporter sets $(P<0 \cdot 01$; Fig. 2A and B). FOXF2 and NHLH1 motifs were exclusive to those reporters strongly affected and SOX17 to those weakly affected by SGTA (Fig. 2A and $\mathrm{B}$ ). AR was the most similarly enriched motif between the two groups, whereas the overall enrichment of transcription factor binding sites was greater in those most affected by SGTA. The above results suggest that SGTA may disrupt the coordination between AR and other factors in the amplification (or inhibition) of a transcriptional response. Collectively, these data imply that a change in the AR:SGTA ratio may alter the qualitative and quantitative cellular response to androgens, particularly at relatively low intracellular ligand concentrations such as might occur following androgen deprivation in prostate cancer.

\section{Structural integrity of amino acids $1-80$ is required for SGTA homodimerization}

SGTA is unique among the steroid receptor-associated TPR-containing co-chaperones in its ability to form homodimers. Although we and others have previously demonstrated that this process relies on the first 80 amino acids of SGTA (Liou \& Wang 2005, Buchanan et al. 2007), the contribution of individual amino acid sequences to the formation of SGTA homodimers as well as the overall importance of SGTA homodimerization to AR signaling still remain unknown. To more precisely map this domain, we constructed a series of variants deleted of 20 amino acid segments within the first 80 residues (i.e. $\Delta 1-20, \Delta 21-40, \Delta 41-60$, and $\Delta 61-80$; Fig. 3A). Unexpectedly, each deletion abolished homodimerization to the same extent as deletion of the full 80 residues (Fig. 3B). Increasing the DNA concentration of any of the deletion constructs failed to rescue SGTA homodimerization (Fig. 3C). Abrogation of SGTA homodimerization was not due to a loss of protein expression (Fig. 3C), but the observed decrease in steady state levels implies that SGTA homodimer formation contributes to overall protein stability.

In order to identify structural elements contributing to SGTA homodimerization, we applied to the SGTA 1-80 sequence i) multiple sequence alignment for SGTA molecules from nine different species, ii) amino 

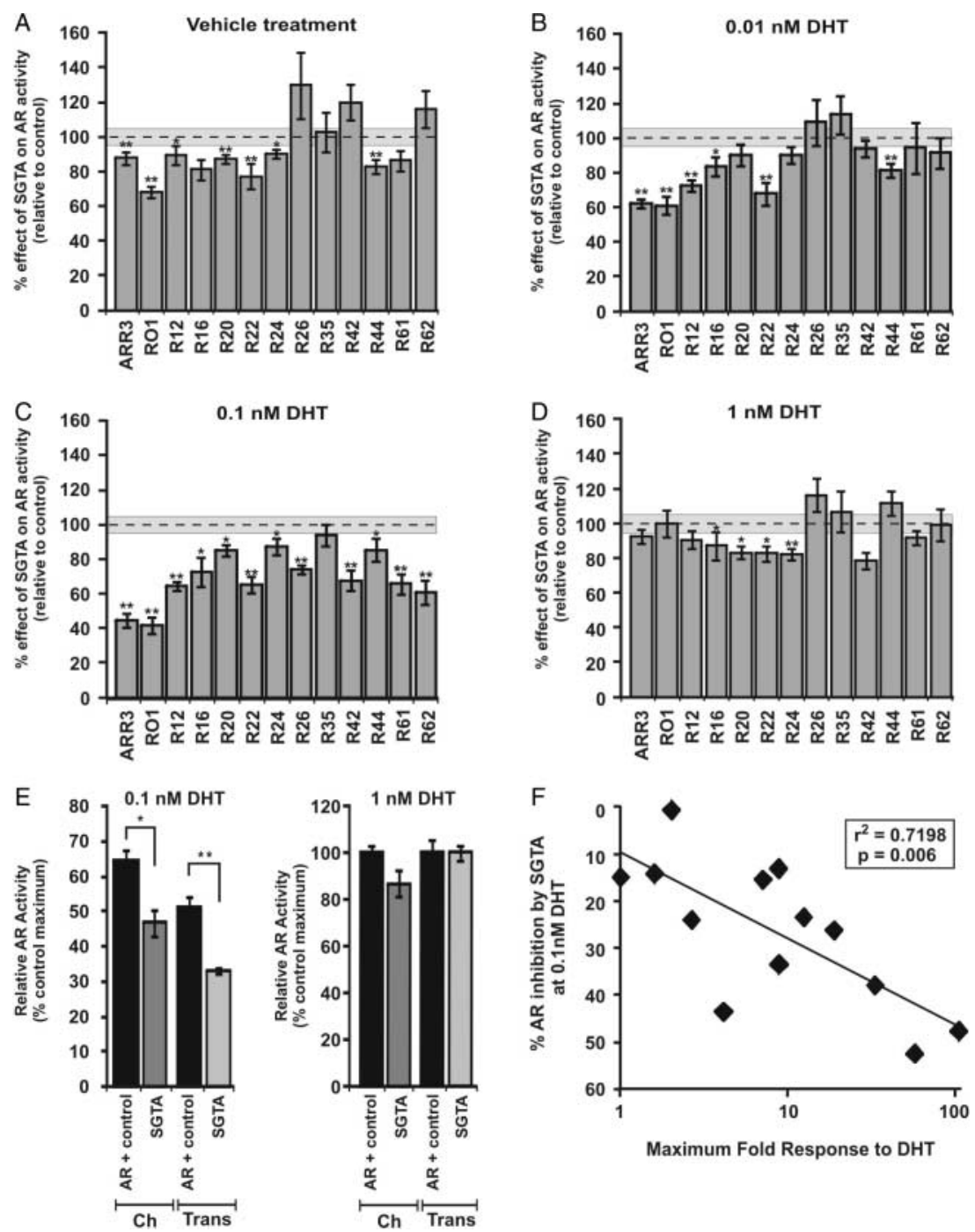

Figure 1 Effect of SGTA on AR signaling on multiple reporter constructs. (A, B, C and D) Effect of SGTA on AR ligand-independent and -dependent transactivation activity. PC3 cells were transfected with AR $(2.5 \mathrm{ng})$, SGTA $(5 \mathrm{ng})$ or empty plasmid control, and one of 13 AR reporter constructs $(50 \mathrm{ng})$ and treated with vehicle (ethanol) or 0.01-1 nM DHT. Data represent combined results from two to five independent experiments (up to 30 replicates/reporter) and are presented as percent ( \pm s.E.M.). Percentage effect of SGTA on $A R$ activity is relative to AR + control (dotted line). Horizontal gray bar at each ligand concentration represents ( \pm median S.E.M.) for $A R+$ control across all reporters.

Significance denoted by ${ }^{\star} P<0.05$, ${ }^{\star} P<0.01$ on Mann-Whitney $U$ for difference between medians of AR + SGTA in comparison to reporter-matched control. (E) Effect of SGTA on AR activation of a chromatin (Ch)-integrated reporter using the PC3 ARR3 stable line compared with transiently transfected (Trans) ARR3 at 0.1 and $1 \mathrm{nM} \mathrm{DHT}$ respectively. ${ }^{\star} P<0.05$, ${ }^{* \star} P<0.01$ on Mann-Whitney $U$ analysis for differences between medians as described in $(A)$. (F) AR transactivation data generated from $C$ were converted and ranked as percentage inhibition of AR activity by SGTA at $0.1 \mathrm{nM} \mathrm{DHT}$. Data represent Spearman $r$ correlation between the percentage inhibition of $A R$ and the maximal activation potential of AR over 0.01-1 nM DHT.

acid composition analysis, and iii) secondary structural prediction. Those analyses revealed enrichment of nonpolar hydrophobic amino acids and alpha helices $(P<0 \cdot 02)$ and a high degree of evolutionary conservation across the entire region, including an uninterrupted stretch of sequence identity from amino acids 27 to 41 (Fig. 3D). Interestingly, the ${ }^{29}$ QESLE $^{33}$ peptide within that region is similar to the AR peptide 


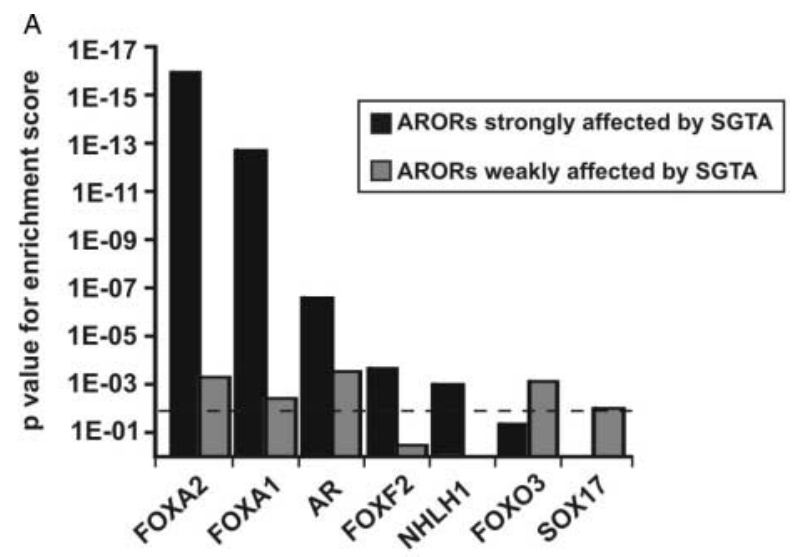

Transcription Factor Binding Sites

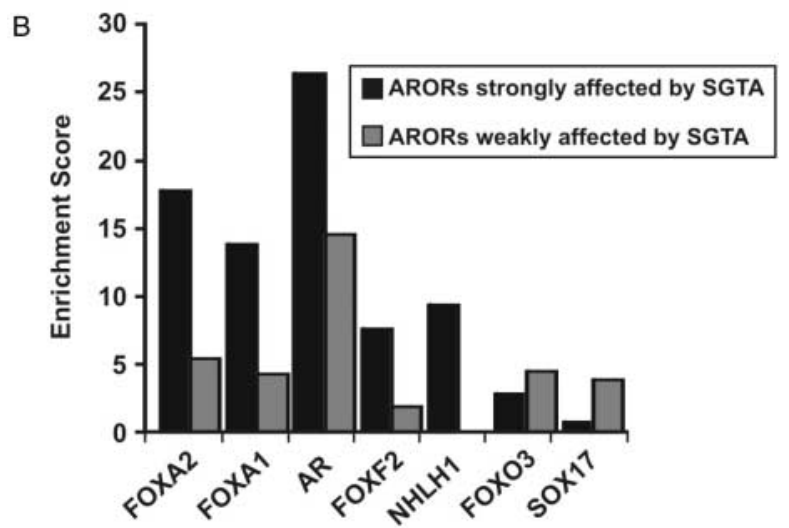

Transcription Factor Binding Sites

Figure 2 Identification of transcription factor motifs in AROR reporters. (A and $B$ ) Androgen receptor reporter constructs were segregated into those that $A R$ activity was strongly affected by SGTA (RO1, R12, R26, R42, and R62) and those that were weakly affected by SGTA (R16, R20, R24, R22, R35, R44, and R61). The JASPAR CORE vertebrata database was used to scan for enrichment of transcription factor binding motifs in both groups. Black bars represent enrichment of transcription factor motifs enriched in reporters strongly affected by SGTA, while gray bars represent enrichment in weakly affected reporters. Significance was determined as $P<0.01$, which is denoted by the dotted line in (A). Enrichment score represents fold enrichment over the background genomic average $(B)$.

${ }^{642}$ QEEGE $^{662}$ previously implicated as mediating the SGTA-AR interaction (Buchanan et al. 2007). When we deleted SGTA of this peptide (i.e. SGTA $\Delta 29-33$ ), mammalian two-hybrid analysis indicated that the variant was incapable of homodimerization, even when we increased the amount of transfected DNA (Fig. 3E).

As TPR domains are major binding sites for client molecules, including HSP90 and HSP70, we next investigated a possible contribution of the TPR domain in the process of SGTA homodimerization. However, the TPR-deleted SGTA variant undergoes homodimerization in an equivalent manner to fulllength SGTA (Fig. 3F). Similar results were obtained for deletion of the QRD with or without the linker region (Fig. 3G). Collectively, these results imply that the presence of conserved amino acids across 1-80 are essential for the structural integrity of the entire domain and thus are indispensable for the maintenance of SGTA homodimerization. In addition, these findings also suggest that the process of SGTA homodimerization may occur independently of the HSP70/90 chaperone complexes.

Finally, we used denaturing and native PAGE analysis to look further at the homodimerization of SGTA and SGTA deletion variants. By standard immunoblot analysis of denatured SGTA proteins, deletion of any amino acid region within the amino terminus had only a minimal effect on SGTA steady state protein levels (Fig. 4A). On nondenaturing, PAGE SGTA bands at 64 and $36 \mathrm{kDa}$ were observed for the full-length protein, which likely represents homodimeric (major proportion) and monomeric (minor proportion) forms of SGTA respectively (Fig. 4B). By contrast, segmented deletions within the homodimerization domain $(\Delta 1-20, \Delta 21-40, \Delta 41-60$, and $\Delta 29-33)$ resulted in a disruption of the observed banding pattern. This did not occur with deletion of the TPR, linker region, or glutamine rich domains. The above data suggest that deletions within the amino terminus of SGTA may lead to a disordered secondary structure and a potential loss in the ability of SGTA to form a homodimer (Fig. 4B).

\section{Amino acids 21-40 of SGTA are necessary for an effect on AR activity}

We next tested whether various homodimerizationdeficient SGTA variants retained their capacity to influence AR function. An SGTA variant without the first 1-80 residues was still capable of repressing AR activity (Fig. 5A). Surprisingly, however, deletion of amino acids 21-40, but not other small sequences within the 1-80 region, abrogated the effect of SGTA on AR activity (Fig. 5A). In order to examine the requirement of other SGTA domains in this process, we generated a series of SGTA constructs deleted of the TPR region the TPR (i.e. $\Delta 91-192$ ) and QRD (i.e. $\Delta 271-313$ ), as well as the interspaced linker region (i.e. $\Delta 200-263$; Fig. 3A). The inhibition of AR activity by SGTA at $0 \cdot 1 \mathrm{nM}$ DHT was unaffected by deletion of the homodimerization domain, partially limited by deletion of the TPR, and abolished for an SGTA variant lacking the linker region $(P<0 \cdot 01$; Fig. 5B). Interestingly, when we tested the effect of SGTA variants on the native AR-responsive reporter R01, the linker deletion variant inhibited AR activity in a manner similar to fulllength SGTA $(P<0 \cdot 05$; Fig. 5C). By contrast, the effect of the $\Delta 21-40$ variant on R01 remained consistent 
A

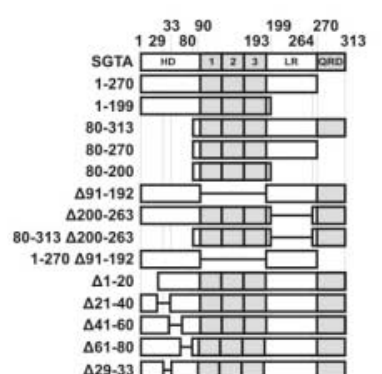

$\Delta 61-80$

$\Delta 29-33$

\section{B}

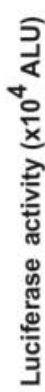

Interaction with SGTA

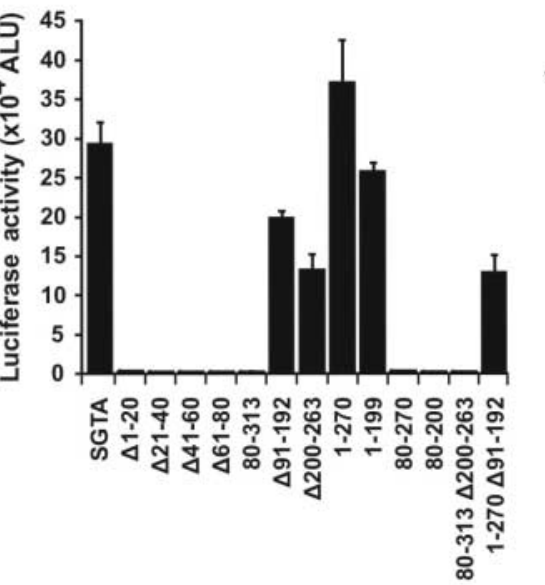

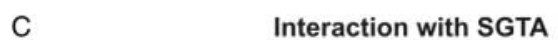

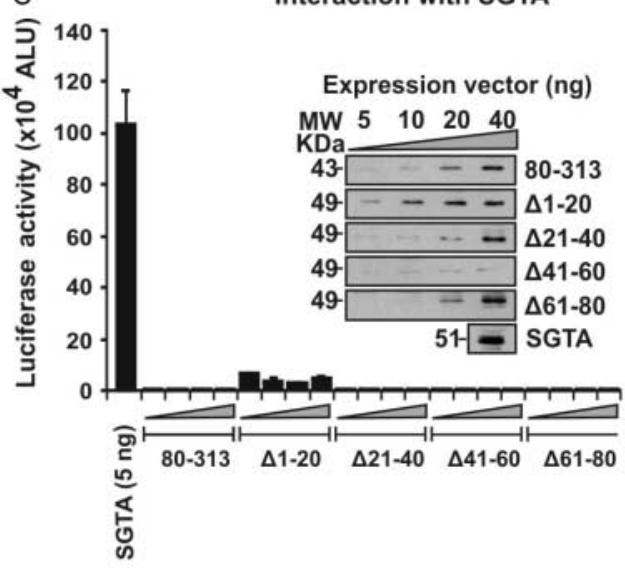

D

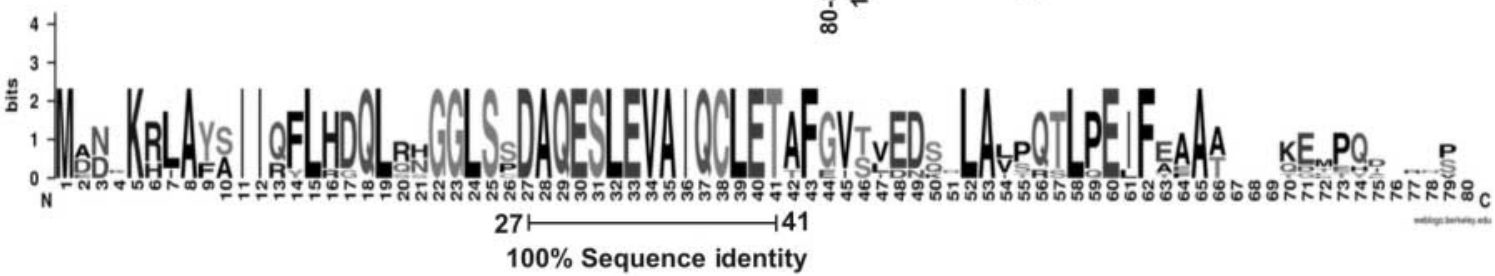

E

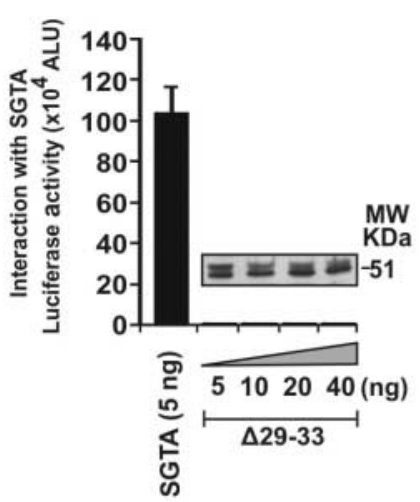

$\mathrm{F}$

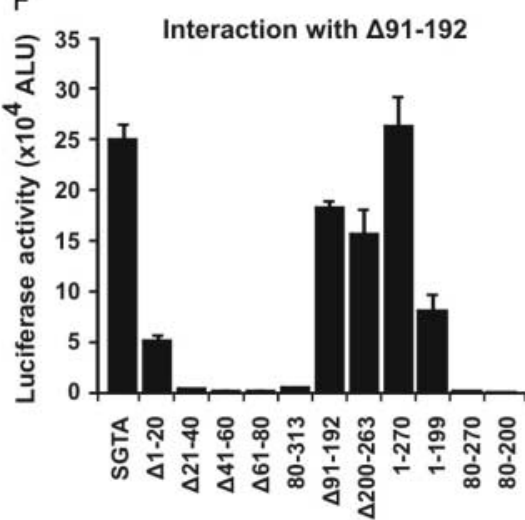

G

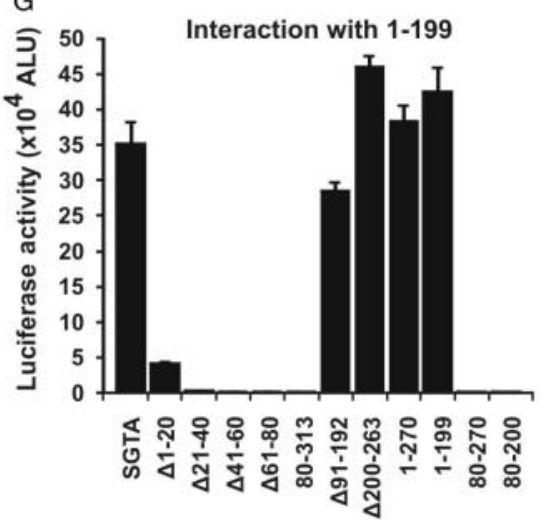

Figure 3 Mapping the SGTA homodimerization surface. (A) SGTA deletion constructs used in this study: 80-313, $\Delta 91-192$, $\Delta 200-263$, and 1-270 represent deletions of the homodimerization (HD), TPR $(1,2,3)$, linker region (LR), and glutamine-rich (QRD) domains respectively. (B, C, E, F and G) Mammalian two-hybrid assays in COS-1 cells co-transfected with 5-40 ng expression plasmids (or other concentrations where indicated) for full-length SGTA and SGTA deletion. Data represent mean ( \pm S.E.M.) arbitrary light units from six independently transfected wells. All data are representative of at least three independently performed experiments. (B) Effect of SGTA deletion on SGTA homodimerization. (C and E) Effect of increasing molar amounts of SGTA deletion variants on SGTA homodimerization. Inset shows immunoblot analysis of SGTA deletion variants. (D) Amino acid sequence logo of the SGTA 1-80 region from nine different species. At each position, height of symbols indicates relative frequency and overall height indicates sequence conservation. Amino acids $27-41$ exhibited $100 \%$ sequence identity across species. ( $F$ and $G$ ) Deletion of the central TPR domain $(\Delta 91-192)$ or the linker and QRD (1-199) does not alter the ability of SGTA to form a homodimer.

compared with the ARR3 reporter. To assess this discrepancy further, we expanded our analysis to the remaining 11 AR-responsive reporters. The response of some reporters to SGTA $\Delta 21-40$ and $\Delta 200-263$ was consistent with R01 (i.e. R12, R22, and R35), but overall there was a high degree of variability in the response of individual cloned loci, which likely results from a compound issue of loci-specific SGTA responses described earlier. Nonetheless, averaging across all reporters suggests that SGTA deleted of the linker region (amino acids 200-263) mostly retains its inhibition of AR, whereas deletion of amino acids 21-40 abolished it $(P<0 \cdot 05)$. We next tested this in vivo by transfection of LNCaP cells with SGTA and variant expression was identified by quantitative RT-PCR and increased translation by immunoblot analysis (Fig. 6A). Overexpression of the full-length SGTA resulted in a significant decrease in PSA (KLK3) mRNA expression 


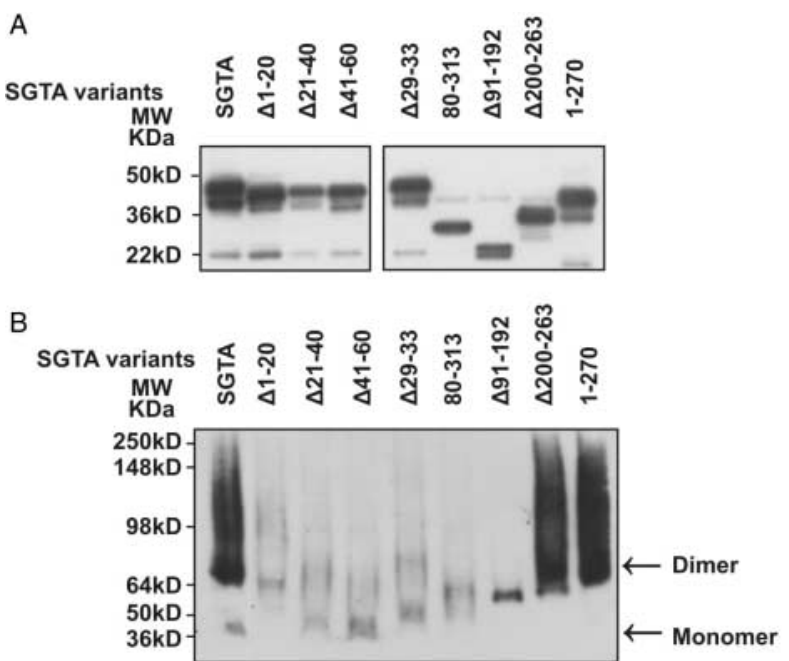

Figure 4 SGTA homodimerization observed by nondenaturing PAGE gel. (A) Denaturing PAGE of SGTA deletion variants expressed in COS-1 cells. (B) Nondenaturing PAGE of same protein lysates as shown in $(A)$.

$(P<0 \cdot 05)$, and a close to significant reduction in FKBP5 $(P=0 \cdot 055$; Fig. $6 \mathrm{~B}$ and $\mathrm{C})$. Importantly, overexpression of the SGTA $\triangle 21-40$ variant had no effect on expression of either PSA or FKBP5 mRNA, whereas SGTA $\triangle 200-263$ significantly reduced expression of both genes $(P<0 \cdot 05$; Fig. $6 \mathrm{~B}$ and $\mathrm{C})$. An important consideration in the above experiment was the concentration of ligand. Previous work in our laboratory showed that ectopic expression of AR in PC3 cells is transcriptionally active at $0 \cdot 1 \mathrm{nM}$ DHT, whereas endogenous $\mathrm{AR}$ in LNCaP cells is activated only at $1 \mathrm{nM}$ DHT (Buchanan et al. 2004a). This explains the effect of SGTA and variants at $1 \mathrm{nM}$ DHT in transfected LNCaP cells, while $0 \cdot 1 \mathrm{nM}$ was the optimal ligand concentration to observe SGTA effects in transfected and highly AR overexpressing PC3 cells. Collectively, the above results suggest that the presence of amino acids 21-40, and not the linker region, is necessary to maintain the inhibitory effects of SGTA on $A R$ transcriptional activity.

\section{Discussion}

The HSP70 and HSP90 molecular chaperone complexes are critical components of steroid receptor signaling by regulating multiple stages of receptor action, including maturation, transport, degradation, and the maintenance of transcriptional activity (Pratt \& Toft 1997). Nonetheless, the mechanisms underpinning chaperone control remain elusive. Emerging evidence for the AR and other steroid receptors suggests that the TPR-containing co-chaperones are dynamic modulators of steroid receptor function
(Smith 2004). Elucidating the function of TPRcontaining proteins is of critical importance in prostate cancer for two key reasons. First, decreased SGTA levels or increased FKBP4, FKBP5, PP5, and/or CYP4O expression have been reported in prostate cancer progression as well as in prostate cancer cell lines compared with normal prostatic tissue and can contribute to enhanced AR function (Buchanan et al. 2007, Periyasamy et al. 2007, 2010). Secondly, a more fully mechanistic understanding is required to appreciate and possibly manipulate the clinical efficacy of new-generation HSP90 inhibitors currently being trialled against solid tumors, including castrate-resistant prostate cancer.

The current study demonstrates that SGTA acts to limit AR responses within a low hormone environment, whereby the predominant effect of SGTA occurs at $\leq 0.1 \mathrm{nM}$ DHT. It is interesting to note that in our previous study (Buchanan et al. 2007), the dynamic range of SGTA inhibition in the presence of DHT appeared to be greater, whereby SGTA was able to restrict $\mathrm{AR}$ activity in ligand concentrations of up to $10 \mathrm{nM}$. We believe that this difference is likely related to the use of a pSG5-based SGTA expression vector, which requires significantly more transfected plasmid, and hence increased squelching of absolute AR activity compared with the high expression of the pCDNA3.1 vector engineered for this study. Nonetheless, the main conclusions from both studies remain highly consistent and valid, i.e. SGTA has a maximal effect on AR activity at $0 \cdot 1 \mathrm{nM}$ DHT. Importantly, the result generated from this work was verified on a representative set of endogenous AR-responsive enhancer loci from chromosomes 20 and 21, which suggests that SGTA plays a relevant biological role in modulating the sensitivity of $A R$ transcriptional activity and decreasing the overall magnitude of androgen signaling in either normal or diseased tissue. Furthermore, the ligand dependency of SGTA action observed here is consistent with the historical perspective of the related co-chaperones (Schulke et al. 2010). However, it has recently become apparent that these diverse molecules also converge on the process of steroid receptor nuclear translocation. The exchange of FKBP5 for FKBP4 or PP5 in the dynein complex facilitates active transport of the holo-AR to the nucleus (Davies et al. 2002). Similarly, inhibition of the dynein-associated co-chaperone Cyp40 blocks AR nuclear translocation in the presence of ligand but has minimal effect on AR ligand binding affinity (Periyasamy et al. 2007). SGTA, which does not exhibit the peptidylprolyl isomerase activity of CYP40, FKBP4, and FKBP5, acts to counter nuclear translocation of the receptor and, consistent with this, decreases the AR transactivation response at $0 \cdot 1 \mathrm{nM}$ DHT, a concentration that would result in $\sim 50 \%$ of receptors being ligand bound (Wilson \& French 1976, 

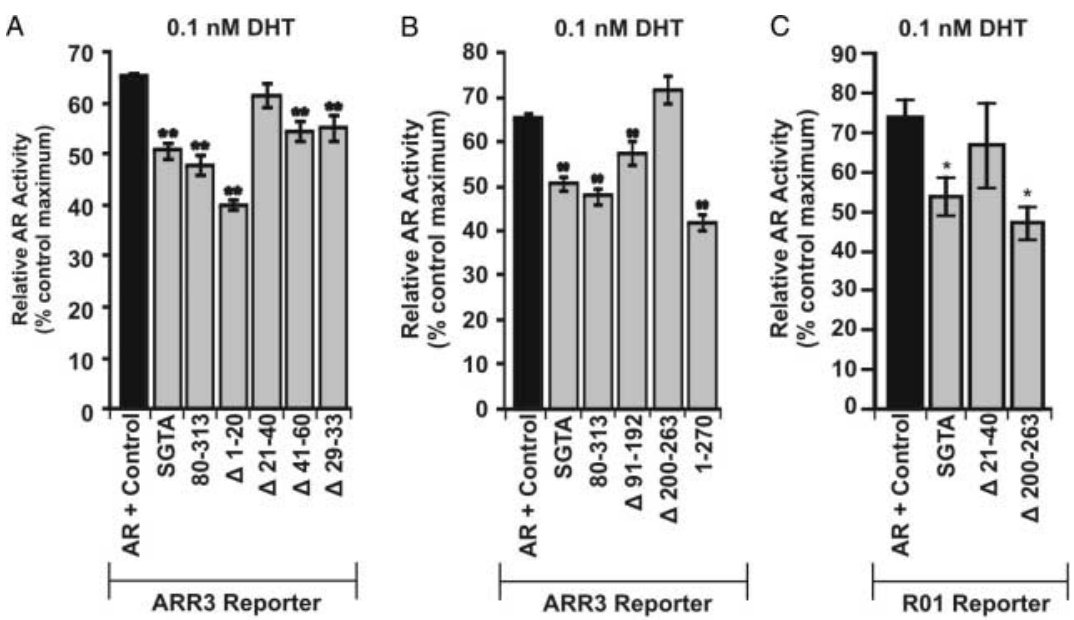

Figure 5 SGTA homodimerization deletion variants variably affect AR activity. (A, B and C) Transactivation activity in transfected PC3 cells of AR at $0.1 \mathrm{nM}$ DHT for $24 \mathrm{~h}$ in the presence of full-length SGTA or homodimerization deletion variants. (A) Effect of SGTA and homodimerization deletion variants on AR activity using the ARR3 reporter. ${ }^{\star \star} P<0 \cdot 01$ on Mann-Whitney $U$ analysis for AR+SGTA and variants compared with AR + control. (B) Transactivation activity of AR in the presence of full-length SGTA or SGTA broad domain deletion variants using the ARR3 reporter. ${ }^{\star \star} P<0.01$ on Mann-Whitney $U$ analysis of medians for AR+SGTA compared with AR+ control. Data are representative of at least three independently performed experiments. (C) Effect of SGTA variants $\Delta 21-40$ and $\Delta 200-263$ on AR activity using the R01 reporter. ${ }^{*} P<0.05$ Data are as presented in (B).

Buchanan et al. 2007). Nonetheless, the results presented here demonstrate that SGTA optimally constrains the sensitivity of the strongest $A R$ transcriptional responses and suggests that SGTA alters AR ligand activation rather than having a direct effect on the general transcriptional machinery. Although it is difficult to decipher the underlying mechanisms surrounding the effects of SGTA on AR activity at different responsive loci, we can speculate that particular DNA sequences and/or additional cofactors that associate with those binding sites may dictate both AR activity and the effectiveness of SGTA-mediated inhibition of AR. Indeed, our bioinformatical analysis of the different reporter constructs revealed enrichment of FOX transcription factor binding motifs in those sites that responded strongly to SGTA compared with those that did not. Moreover, we demonstrated in this study that the inhibitory effect of SGTA is greatly diminished on those DNA binding sites that are least responsive to AR occupancy, suggesting coordination between $A R$ and other transcription factors at these sites to form an active transcriptional complex. Indeed, this differential effect was also observed with our SGTA deletion variants, which suggests a functional response that depends on both the SGTA residues present and the specific DNA target sequence. However, in order to elucidate this mechanism, analysis of SGTA action on genome-wide AR activity would be required. Nevertheless, these findings provide important new information regarding the biological actions of SGTA, which collectively suggest that SGTA likely acts as an important buffer against untoward AR responses, by excluding the AR from the nucleus in the absence of ligand or in the presence of low androgen levels, such as those seen in prostate cancer during the initial stages of androgen deprivation therapy (Feldman \& Feldman 2001, Scher et al. 2004, Scher \& Sawyers 2005). The failure of androgen deprivation may be explained in part by permissive changes in androgen action, including increased AR expression and/or selection of a hypersensitive response. Accordingly, it has been recently reported that intracellular conversion of adrenal androgens and de novo androgen synthesis in prostate cancer compared with normal cells significantly contributes to continued AR activity during the progression to a castrate-resistant diseased state (Montgomery et al. 2008, Knudsen \& Penning 2010). In that context, the accumulation of intracellular androgens would be predicted to override the SGTA effect, which would complement the lower levels of SGTA in prostate cancer cells to favor continued AR signaling in a castrate environment (Scher et al. 2004, Scher \& Sawyers 2005, Hendriksen et al. 2006, Buchanan et al. 2007, Chmelar et al. 2007, Wang et al. 2009). The clinical success of androgen biosynthesis inhibitors, such as abiraterone acetate, may be due in part to reducing intracellular hormone concentrations to a level at which SGTA can prevent AR nuclear transfer (Attard et al. 2008, 2011, Danila et al. 2010).

The ability of SGTA to form a homodimer is unique among the steroid receptor-associated TPR co-chaperones. Although SGTA homodimerization is able to decrease the binding affinity between the TPR domain and the human GHR, the biological function of SGTA homodimerization remains unclear as it is not required for an effect of SGTA on the ATPase activity of HSC70 

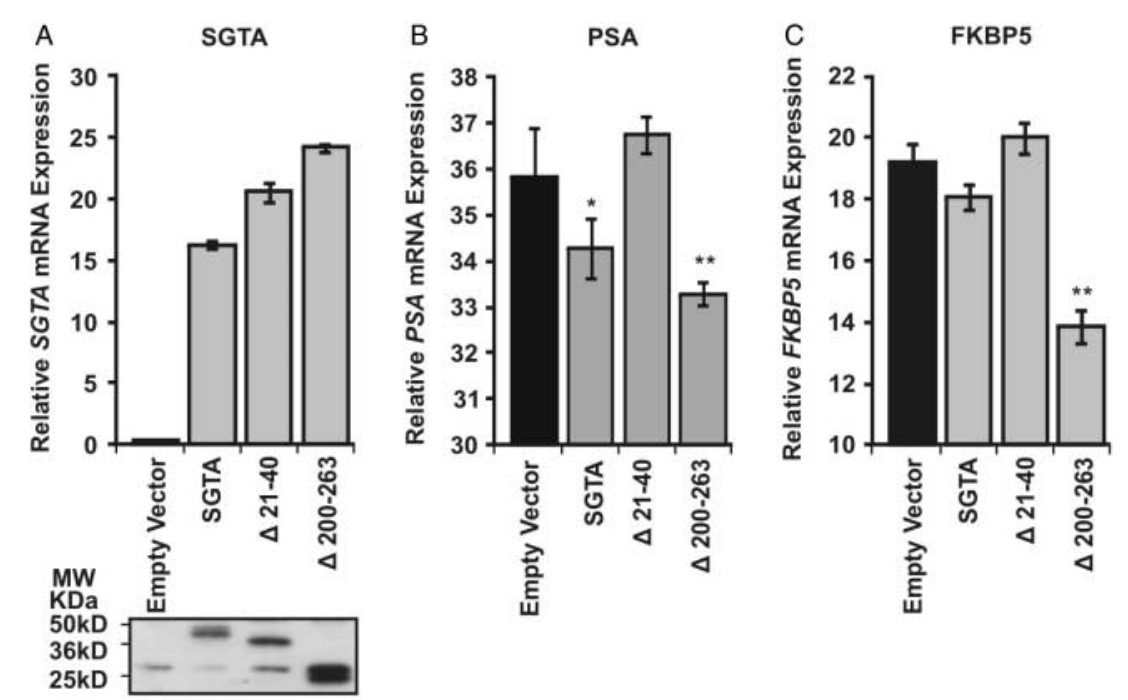

Figure 6 (A, B and C) Effect of SGTA deletion variants $\Delta 21-40$ and $\Delta 200-263$ on endogenous AR activity. LNCaP cells were transfected with either pCFH empty vector, pCFH-SGTA, pCFH-SGTA $\Delta 21-40$, or pCFH-SGTA $\Delta 200-263$. Cells were treated with $1 \mathrm{nM} \mathrm{DHT}$ for $16 \mathrm{~h}$. RNA was isolated and analyzed by quantitative RT-PCR for expression of SGTA, PSA, and FKBP51 and normalized to the housekeeping gene GAPDH. The presented data represent the mean ( \pm S.E.M.) of three biological replicates. Protein lysates were also collected and analyzed by western blot using SGTA antisera. ${ }^{*} P<0.05$,

${ }^{\star *} P<0.01$ on Mann-Whitney $U$ analysis of medians for SGTA or deletion variants compared with pCFH control.

(HSPA8; Liu et al. 1999, Schantl et al. 2003), nor here on AR responses. Nevertheless, this study reveals for the first time that a highly ordered secondary structure within the entire first 80 amino acids likely creates a complex binding surface critical for the formation of SGTA homodimerization as well as maintaining overall protein stability. Moreover, it was determined that the SGTA homodimerization domain was not required for an effect on AR activity, although it was found that deletion of amino acids 21-40 abrogated the inhibitory effect of SGTA. Even though the underlying mechanism remains unclear, given the high degree of conservation between amino acids $27-41$, the effect of the $\Delta 21-40$ SGTA variant on AR activity may be a result of destabilization within the SGTA protein structure, leading to a loss of interaction between SGTA and AR. Although further experiments are required to precisely map the regions of SGTA that interact with AR, this study demonstrated for the first time that deletion of the TPR domain, which is known to be the main interaction surface for HSP70, HSP90, and other client proteins (Angeletti et al. 2002, Yin et al. 2006), did not affect the capacity of SGTA to inhibit AR activity, suggesting that the amino acids required for interaction between SGTA and AR lie outside of the TPR domain. Undoubtedly, complex inter- or intramolecular interactions of SGTA and other TPR chaperones are critical for their capacity to modulate steroid receptor responses.

\section{Conclusion}

In summary, this study not only highlights the complexity surrounding co-chaperone action in steroid receptor signaling but also has firmly demonstrated that the formation of SGTA homodimerization is reliant on the structural integrity of the first 80 amino acids. Interestingly, the biological actions of SGTA to desensitize the activity of AR in the presence of low ligand concentrations appear to be dependent upon a conserved region within the SGTA homodimerization domain. Furthermore, the ability of SGTA to decrease the ligand sensitivity of AR and its activity on highly responsive loci enriched with multiple transcription factor binding sites may be an important mechanism in limiting spurious androgenic responses.

\section{Declaration of interest}

The authors declare that there is no conflict of interest that could be perceived as prejudicing the impartiality of the research reported.

\section{Funding}

Prostate Cancer Foundation of Australia (G B, ID\#YI02 and L A S, ID\#YI0810), The National Health and Medical Research Council of Australia (W D T and L M B, ID\#453662), U.S. Department of Defense (W D T and L M B, ID\#PC060443), HIH/NCI (G A C, R01CA109147 and R01CA136924), and The Prostate Cancer Foundation (G A C). 
G B holds an NHMRC Career Development Award Level 1 (ID\#627018). E F N holds a Freemasons Foundation Postdoctoral Fellowship. L M B holds a Cancer Council South Australia Senior Research Fellowship. L A S holds a Prostate Cancer Foundation Young Investigator award. A P T holds a University of Adelaide Faculty of Health Science PhD Division/Freemasons Foundation Centre for Men's Health Postgraduate Scholarship.

\section{Acknowledgements}

The authors would like to acknowledge Drs Li Jia and Baruch Frenkel for their contribution to the AR luciferase reporter constructs and Dr Jennifer Prescott, Ms Joanna Gillis, Ms Miriam Butler, and Dr Jeff Barrett for their technical assistance.

\section{References}

Angeletti PC, Walker D \& Panganiban AT 2002 Small glutamine-rich protein/viral protein U-binding protein is a novel cochaperone that affects heat shock protein 70 activity. Cell Stress $\mathcal{E}$ Chaperones 7 258-268. (doi:10.1379/1466-1268(2002) 007<0258:SGRPVP > 2.0. $\mathrm{CO} ; 2)$

Attard G, Reid AH, Yap TA, Raynaud F, Dowsett M, Settatree S, Barrett M, Parker C, Martins V, Folkerd E et al. 2008 Phase I clinical trial of a selective inhibitor of CYP17, abiraterone acetate, confirms that castration-resistant prostate cancer commonly remains hormone driven. Journal of Clinical Oncology 26 4563-4571. (doi:10.1200/JCO.2007.15.9749)

Attard G, Richards J \& de Bono JS 2011 Targeting the androgen receptor signaling pathway in metastatic prostate cancer. Clinical Cancer Research 17 1649-1657. (doi:10.1158/1078-0432)

Barent RL, Nair SC, Carr DC, Ruan Y, Rimerman RA, Fulton J, Zhang Y \& Smith DF 1998 Analysis of FKBP51/FKBP52 chimeras and mutants for Hsp90 binding and association with progesterone receptor complexes. Molecular Endocrinology 12 342-354. (doi:10.1210/me.12.3.342)

Blom N, Gammeltoft S \& Brunak S 1999 Sequence and structure-based prediction of eukaryotic protein phosphorylation sites. Journal of Molecular Biology 294 1351-1362. (doi:10.1006/jmbi.1999.3310)

Bryne JC, Valen E, Tang MH, Marstrand T, Winther O, da Piedade I, Krogh A, Lenhard B \& Sandelin A 2008 JASPAR, the open access database of transcription factor-binding profiles: new content and tools in the 2008 update. Nucleic Acids Research 36 D102-D106. (doi:10.1093/nar/gkm955)

Buchanan G, Craft PS, Yang M, Cheong A, Prescott J, Jia L, Coetzee GA \& Tilley WD 2004 $a$ PC-3 cells with enhanced androgen receptor signaling: a model for clonal selection in prostate cancer. Prostate 60 352-366. (doi:10.1002/pros.20079)

Buchanan G, Yang M, Cheong A, Harris JM, Irvine RA, Lambert PF, Moore NL, Raynor M, Neufing PJ, Coetzee GA et al. 2004b Structural and functional consequences of glutamine tract variation in the androgen receptor. Human Molecular Genetics 13 1677-1692. (doi:10.1093/hmg/ddh181)

Buchanan G, Ricciardelli C, Harris JM, Prescott J, Yu ZC, Jia L, Butler LM, Marshall VR, Scher HI, Gerald WL et al. 2007 Control of androgen receptor signaling in prostate cancer by the cochaperone small glutamine rich tetratricopeptide repeat containing protein alpha. Cancer Research 67 10087-10096. (doi:10.1158/0008-5472.CAN-07-1646)

Butler LM, Centenera MM, Neufing PJ, Buchanan G, Choong CS, Ricciardelli C, Saint K, Lee M, Ochnik A, Yang M et al. 2006 Suppression of androgen receptor signaling in prostate cancer cells by an inhibitory receptor variant. Molecular Endocrinology 20 1009-1024. (doi:10.1210/me.2004-0401)
Cheung-Flynn J, Prapapanich V, Cox MB, Riggs DL, Suarez-Quian C \& Smith DF 2005 Physiological role for the cochaperone FKBP52 in androgen receptor signaling. Molecular Endocrinology 19 1654-1666. (doi:10.1210/me.2005-0071)

Chmelar R, Buchanan G, Need EF, Tilley W \& Greenberg NM 2007 Androgen receptor coregulators and their involvement in the development and progression of prostate cancer. International Journal of Cancer 120 719-733. (doi:10.1002/ijc.22365)

Cziepluch C, Kordes E, Poirey R, Grewenig A, Rommelaere J \& Jauniaux JC 1998 Identification of a novel cellular TPR-containing protein, SGT, that interacts with the nonstructural protein NS1 of parvovirus H-1. Journal of Virology 72 4149-4156.

Cziepluch C, Lampel S, Grewenig A, Grund C, Lichter P \& Rommelaere J $2000 \mathrm{H}-1$ parvovirus-associated replication bodies: a distinct virus-induced nuclear structure. Journal of Virology $\mathbf{7 4}$ 4807-4815. (doi:10.1128/JVI.74.10.4807-4815.2000)

Danila DC, Morris MJ, de Bono JS, Ryan CJ, Denmeade SR, Smith MR, Taplin ME, Bubley GJ, Kheoh T, Haqq C et al. 2010 Phase II multicenter study of abiraterone acetate plus prednisone therapy in patients with docetaxel-treated castration-resistant prostate cancer. Journal of Clinical Oncology 28 1496-1501. (doi:10.1200/JCO.2009. 25.9259)

Davies TH, Ning YM \& Sanchez ER 2002 A new first step in activation of steroid receptors: hormone-induced switching of FKBP51 and FKBP52 immunophilins. Journal of Biological Chemistry 277 4597-4600. (doi:10.1074/jbc.C100531200)

Denny WB, Valentine DL, Reynolds PD, Smith DF \& Scammell JG 2000 Squirrel monkey immunophilin FKBP51 is a potent inhibitor of glucocorticoid receptor binding. Endocrinology 141 4107-4113. (doi:10.1210/en.141.11.4107)

Feldman BJ \& Feldman D 2001 The development of androgenindependent prostate cancer. Nature Reviews. Cancer $134-45$. (doi:10.1038/35094009)

Hendriksen PJ, Dits NF, Kokame K, Veldhoven A, van Weerden WM, Bangma CH, Trapman J \& Jenster G 2006 Evolution of the androgen receptor pathway during progression of prostate cancer. Cancer Research 66 5012-5020. (doi:10.1158/0008-5472. CAN-05-3082)

Ji H, Jiang H, Ma W, Johnson DS, Myers RM \& Wong WH 2008 An integrated software system for analyzing ChIP-chip and ChIP-seq data. Nature Biotechnology 26 1293-1300. (doi:10.1038/nbt.1505)

Jia L, Berman BP, Jariwala U, Yan X, Cogan JP, Walters A, Chen T, Buchanan G, Frenkel B \& Coetzee GA 2008 Genomic androgen receptor-occupied regions with different functions, defined by histone acetylation, coregulators and transcriptional capacity. PLoS ONE 3 e3645. (doi:10.1371/journal.pone.0003645)

Katzman S, Barrett C, Thiltgen G, Karchin R \& Karplus K 2008 PREDICT-2ND: a tool for generalized protein local structure prediction. Bioinformatics 24 2453-2459. (doi:10.1093/bioinformatics/btn438)

Knudsen KE \& Penning TM 2010 Partners in crime: deregulation of AR activity and androgen synthesis in prostate cancer. Trends in Endocrinology and Metabolism 21 315-324. (doi:10.1016/j.tem.2010. 01.002)

Liou ST \& Wang C 2005 Small glutamine-rich tetratricopeptide repeatcontaining protein is composed of three structural units with distinct functions. Archives of Biochemistry and Biophysics 435 253-263. (doi:10.1016/j.abb.2004.12.020)

Liu FH, Wu SJ, Hu SM, Hsiao CD \& Wang C 1999 Specific interaction of the $70-\mathrm{kDa}$ heat shock cognate protein with the tetratricopeptide repeats. Journal of Biological Chemistry 274 34425-34432. (doi:10.1074/jbc.274.48.34425)

Marcelli M, Stenoien DL, Szafran AT, Simeoni S, Agoulnik IU, Weigel NL, Moran T, Mikic I, Price JH \& Mancini MA 2006 Quantifying effects of ligands on androgen receptor nuclear translocation, intranuclear dynamics, and solubility. Journal of Cellular Biochemistry 98 770-788. (doi:10.1002/jcb.20593) 
Montgomery RB, Mostaghel EA, Vessella R, Hess DL, Kalhorn TF, Higano CS, True LD \& Nelson PS 2008 Maintenance of intratumoral androgens in metastatic prostate cancer: a mechanism for castration-resistant tumor growth. Cancer Research 68 4447-4454. (doi:10.1158/0008-5472.CAN-08-0249)

Need EF, Scher HI, Peters AA, Moore NL, Cheong A, Ryan CJ, Wittert GA, Marshall VR, Tilley WD \& Buchanan G 2009 A novel androgen receptor amino terminal region reveals two classes of amino/carboxyl interaction-deficient variants with divergent capacity to activate responsive sites in chromatin. Endocrinology 150 2674-2682. (doi:10.1210/en.2008-1181)

Periyasamy S, Warrier M, Tillekeratne MP, Shou W \& Sanchez ER 2007 The immunophilin ligands cyclosporin A and FK506 suppress prostate cancer cell growth by androgen receptor-dependent and -independent mechanisms. Endocrinology 148 4716-4726. (doi:10.1210/en.2007-0145)

Periyasamy S, Hinds T Jr, Shemshedini L, Shou W \& Sanchez ER 2010 FKBP51 and Cyp40 are positive regulators of androgen-dependent prostate cancer cell growth and the targets of FK506 and cyclosporin A. Oncogene 29 1691-1701. (doi:10.1038/onc.2009.458)

Pratt WB \& Toft DO 1997 Steroid receptor interactions with heat shock protein and immunophilin chaperones. Endocrine Reviews 18 306-360. (doi:10.1210/er.18.3.306)

Schantl JA, Roza M, De Jong AP \& Strous GJ 2003 Small glutamine-rich tetratricopeptide repeat-containing protein (SGT) interacts with the ubiquitin-dependent endocytosis (UbE) motif of the growth hormone receptor. Biochemical Journal 373 855-863. (doi:10.1042/ BJ20021591)

Scher HI \& Sawyers CL 2005 Biology of progressive, castration-resistant prostate cancer: directed therapies targeting the androgenreceptor signaling axis. Journal of Clinical Oncology 23 8253-8261. (doi:10.1200/JCO.2005.03.4777)

Scher HI, Buchanan G, Gerald W, Butler LM \& Tilley WD 2004 Targeting the androgen receptor: improving outcomes for castration-resistant prostate cancer. Endocrine-Related Cancer 11 459-476. (doi:10.1677/erc.1.00525)

Schulke JP, Wochnik GM, Lang-Rollin I, Gassen NC, Knapp RT, Berning B, Yassouridis A \& Rein T 2010 Differential impact of tetratricopeptide repeat proteins on the steroid hormone receptors. PLoS ONE 5 e11717. (doi:10.1371/journal.pone. 0011717)

Smith DF 2004 Tetratricopeptide repeat cochaperones in steroid receptor complexes. Cell Stress $\mathcal{E} \odot$ Chaperones 9 109-121. (doi:10.1379/CSC-31.1)

Sun K, Montana V, Chellappa K, Brelivet Y, Moras D, Maeda Y, Parpura V, Paschal BM \& Sladek FM 2007 Phosphorylation of a conserved serine in the deoxyribonucleic acid binding domain of nuclear receptors alters intracellular localization. Molecular Endocrinology 21 1297-1311. (doi:10.1210/me.2006-0300)

Thomas M, Harrell JM, Morishima Y, Peng HM, Pratt WB \& Lieberman AP 2006 Pharmacologic and genetic inhibition of hsp90-dependent trafficking reduces aggregation and promotes degradation of the expanded glutamine androgen receptor without stress protein induction. Human Molecular Genetics 15 1876-1883. (doi:10.1093/hmg/dd1110)

Tobaben S, Varoqueaux F, Brose N, Stahl B \& Meyer G 2003 A brain-specific isoform of small glutamine-rich tetratricopeptide repeat-containing protein binds to $\mathrm{Hsc70}$ and the cysteine string protein. Journal of Biological Chemistry 278 38376-38383. (doi:10.1074/jbc.M301558200)

Vacic V, Uversky VN, Dunker AK \& Lonardi S 2007 Composition Profiler: a tool for discovery and visualization of amino acid composition differences. BMC Bioinformatics 8 211. (doi:10.1186/ 1471-2105-8-211)

Wang H, Zhang Q \& Zhu D 2003 hSGT interacts with the N-terminal region of myostatin. Biochemical and Biophysical Research Communications 311 877-883. (doi:10.1016/j.bbrc.2003.10.080)

Wang Q, Li W, Zhang Y, Yuan X, Xu K, Yu J, Chen Z, Beroukhim R, Wang H, Lupien M et al. 2009 Androgen receptor regulates a distinct transcription program in androgen-independent prostate cancer. Cell 138 245-256. (doi:10.1016/j.cell.2009.04.056)

Wilson EM \& French FS 1976 Binding properties of androgen receptors. Evidence for identical receptors in rat testis, epididymis, and prostate. Journal of Biological Chemistry 251 5620-5629.

Winnefeld M, Rommelaere J \& Cziepluch C 2004 The human small glutamine-rich TPR-containing protein is required for progress through cell division. Experimental Cell Research 293 43-57. (doi:10.1016/j.yexcr.2003.09.028)

Winnefeld M, Grewenig A, Schnolzer M, Spring H, Knoch TA, Gan EC, Rommelaere J \& Cziepluch C 2006 Human SGT interacts with Bag-6/Bat-3/Scythe and cells with reduced levels of either protein display persistence of few misaligned chromosomes and mitotic arrest. Experimental Cell Research 312 2500-2514. (doi:10.1016/ j.yexcr.2006.04.020)

Yin H, Wang H, Zong H, Chen X, Wang Y, Yun X, Wu Y, Wang J \& Gu J 2006 SGT, a Hsp90beta binding partner, is accumulated in the nucleus during cell apoptosis. Biochemical and Biophysical Research Communications 343 1153-1158. (doi:10.1016/j.bbrc.2006.03.090)

Yong W, Yang Z, Periyasamy S, Chen H, Yucel S, Li W, Lin LY, Wolf IM, Cohn MJ, Baskin LS et al. 2007 Essential role for co-chaperone Fkbp52 but not Fkbp51 in androgen receptor-mediated signaling and physiology. Journal of Biological Chemistry 282 5026-5036. (doi:10.1074/jbc.M609360200)

Zhang L, Johnson M, Le KH, Sato M, Ilagan R, Iyer M, Gambhir SS, Wu L \& Carey M 2003 Interrogating androgen receptor function in recurrent prostate cancer. Cancer Research 63 4552-4560.

Ziegler EC \& Ghosh S 2005 Regulating inducible transcription through controlled localization. Science's STKE 2005 re6. (doi:10.1126/stke.2842005re6)

Received in final form 25 May 2012

Accepted 8 June 2012

Made available online as an Accepted Preprint 12 June 2012 